\title{
Mobility Load Balancing in Cellular System with Multicriteria Handoff Algorithm
}

\author{
Solomon T. Girma ${ }^{1}$ and Abinet G. Abebe ${ }^{2}$ \\ ${ }^{1}$ Department of Electrical Engineering, Pan African University Institute of Sciences, Technology and Innovations, \\ Addis Ababa, Ethiopia \\ ${ }^{2}$ Ethio Telecom, Radio Access Network Rollout Department, Addis Ababa, Ethiopia
}

Correspondence should be addressed to Solomon T. Girma; solomon.tshm@gmail.com

Received 18 June 2017; Accepted 18 October 2017; Published 29 November 2017

Academic Editor: Antonella Petrillo

Copyright (C) 2017 Solomon T. Girma and Abinet G. Abebe. This is an open access article distributed under the Creative Commons Attribution License, which permits unrestricted use, distribution, and reproduction in any medium, provided the original work is properly cited.

\begin{abstract}
Efficient traffic load balancing algorithm is very important to serve more mobile users in the cellular networks. This paper is based on mobility load balancing handoff algorithm using fuzzy logic. The rank of the serving and the neighboring Base Transceiver Stations (BTSs) are calculated every half second with the help of measurement report from the two-ray propagation model. This algorithm is able to balance load of the BTS by handing off some ongoing calls on BTS's edge of highly loaded BTS to move to overlapping underloaded BTS, such that the coverage area of loaded BTS virtually shrunk towards BTS center of a loaded sector. In case of low load scenarios, the coverage area of a BTS is presumed to be virtually widened to cover up to the partial serving area of neighboring BTS. This helps a highly loaded neighboring BTS or failed BTS due to power or transmission. Simulation shows that new call blocking and handoff blocking using the proposed algorithm are enhanced notably.
\end{abstract}

\section{Introduction}

Load balancing is a mechanism whereby overloaded BTSs distribute some of their traffic to less loaded neighbors in order to make the radio resource more efficient [1].

Telecommunication infrastructures originally designed to carry a defined amount of traffic are often congested by an overwhelming request of resources. A naive solution is to expand the infrastructure to match the increasing demand. Due to limitations in available space and shortness of resources, this solution is not possible. The best solution is to carefully tune the parameters of the existing system to accommodate the new traffic demands [2].

There are a number of methods to balance traffic load among BTS [3]. One of the most important methods is based on antenna down tilting [4]. With the down tilt, one directs the antenna radiation further down to the ground. The down tilt is advisable when one wishes to decrease interference and coverage in some specific areas, each BTS to meet only its designed area. When selecting the optimum tilt angle, the goal is to have as high signal strength as possible in the area where the BTS should be serving traffic [4]. Beyond the serving area of the BTS, the signal strength should be as low as possible. A too aggressive down tilting strategy however leads to an overall loss of coverage and creates coverage holes which eventually lead to call drop.

The second method is to adjust the BTS coverage by varying transmitter power. A minimal transmitter power effectively disallows more distant mobiles to access the BTS, thereby decreasing the coverage area and prohibiting distant mobile users to access the BTS [5]. Hence, reducing BTS coverage area runs at the danger of creating coverage holes. Having the coverage holes on the cellular system adversely affect the performance of cellular system leading to call drop. Conversely maximum transmitter power effectively allows mobile at distance to access the BTS, at the cost of producing interference on those BTSs using the same frequency [5].

The antenna height is fundamental to BTS coverage area. If the antenna height is increased, path-loss will reduce. The relation between antenna height and coverage area is stated on two-ray model [6]. If antenna height is doubled, then coverage will be increased by $6 \mathrm{db}[6]$. To prohibit the 
distant mobile user from accessing BTS, reducing the height of antenna is good alternative.

Mobile station (MS), in the presence of random networks with overlapping cellular coverage, can connect to any of BTSs. MS in a wireless network switches its current Point of Attachment (PoA) to a new wireless network using a process called handoff [7]. To have global connectivity, handoffs are extremely important cellular communication because of the cellular architecture employed to maximize spectrum utilization. When a mobile terminal moves away from a base station, the signal level drops and there is a need to switch the communications channel to another base station. That time there is a need for a handoff to be executed. Handoff is the process of changing the communications channel associated with the current ongoing connection while a call is in progress [8].

Therefore, by adjusting the handoff regions between neighboring BTSs, it is possible to cause cell edge users in overloaded BTS to migrate to less loaded neighboring BTS. Such an approach is referred to as mobility load balancing, thereby increasing the efficiency of resource utilization [9].

Many metrics have been used to support handoff decisions, including received signal strength (RSS), signal to noise ratio (SIR), power budget, and distance between the mobile station and BTS, traffic load, and mobile velocity, among others. The single criteria handoff decision compares one of the metrics from the serving BTS with that from one of the neighboring BTSs, using a constant handoff threshold value. The selection of the threshold is important to handoff performance. If the threshold is too small, a lot of unnecessary handoffs may take place. On the contrary, the quality of service (QoS) could be low and calls could be dropped if the threshold is too large.

However, all the above techniques of load balancing are not self-adaptive. The problem of network congestion control remains a critical issue and a high priority, mainly given the growing size, demand, and speed of the networks. Therefore, network congestion is becoming a real threat to the growth of existing real time networks (circuit switching). It is a problem that cannot be ignored.

A multicriteria handoff algorithm can provide better performance than a single criterion handoff algorithm due to the extra number of evaluation parameters and the greater potential for achieving the desired balance among different system characteristics. This multicriteria nature of the algorithm allows simultaneous consideration of several significant aspects of the handoff procedure in order to enhance the system performance [8].

Justification of Using Fuzzy Logic in Handoff Process. Network parameters like RSS, power budget, SNR, and traffic load are intrinsically imprecise, vague, and uncertain [10]. Due to this nature of fuzziness, the accurate measurement of these network parameters in a wireless environment is a difficult task. A fuzzy logic method seems to produce better results when used for system design in such condition [10].

The fact that fuzzy logic can mimic human expert reasoning and that many of the terms used to describe a radio signal (weak, far, strong, and congested) are fuzzy in nature makes fuzzy logic a strong candidate for performing handoff decisions. Fuzzy logic can adapt easily to these decisions as it can overcome radio environment uncertainty and fluctuations and can deal with heterogeneous intersystem parameters (shadowing effect, traffic variations, etc.) [11].

\section{System Model}

2.1. Network Model. We consider a GSM cellular network in which cells are omnidirectional that each cell is served by central BTS and has 100 traffic channels. Mobile users inside the coverage area of a BTS are assumed to be log-normal distribution. BTSs are connected to a single BTS Controller (BSC) where the proposed algorithm is to be located. A mobile station measures the received signal strength, the path-loss, and the received signal to interference ratio from the current serving BTS and all the neighboring BTS. This measurement report is done by mobile station every half second and report to BTS through common control channel. The proposed algorithm ranks the index of all the BTSs in the whole cluster and determines if handoff has to take place.

In this paper, we are assuming a suburban area environment. In instances of low load scenarios, the coverage area of a cell sector is assumed to be virtually extendable to cover up to the partial serving area of a nearby BTS when the BTS has low or medium load. This helps a nearby highly loaded or failed BTS due to power or transmission. Figure 1 shows a cluster of seven cells, whereby every BTS has gotten six adjacent cells. In case of congestion, the adjacent cell will cover its immediate cell.

\section{Propagation Model}

The two-ray model is used when a single ground reflection dominates the multipath effect. The received signal consists of two components: the line of sight component which is just the transmitted signal propagating through free space and a reflected component which is the transmitted signal reflected off the ground [6]. Equation (1) indicates the received power falls off inversely the fourth power of $d$ and independent of wavelength.

$$
\begin{aligned}
\operatorname{Pr} \mathrm{dbm}= & \text { pt dbm }+20 \log _{10}(\mathrm{hthr})-40 \log _{10} d+\mathrm{Gr} \\
& +\mathrm{Gt}
\end{aligned}
$$

where $\operatorname{Pr}$ is the received power in $\mathrm{dbm}$, pt is transmitted power in $\mathrm{dbm}, \mathrm{Gr}$ is receiver antenna gain in $\mathrm{db}$, Gt is transmitter antenna gain in $\mathrm{db}$, ht is transmitter height in meters, hr is received antenna height in meters, and $d$ is the distance between transmitter and receiver in meters.

Figure 2 shows the received signal strength (RSS) from two neighboring BTSs. It is assumed that the RSS averaged over time, so the fluctuations due to multipath nature of the radio environment can be eliminated [13]. Figure 2 shows a MS moving from BTS I to BTS J. The RSS of BTS I decrease as MS moves away from the BTS and from BTS J increases as it approaches. With conventional algorithm, looking at the variation of RSS from either BTS; it is possible to tell 


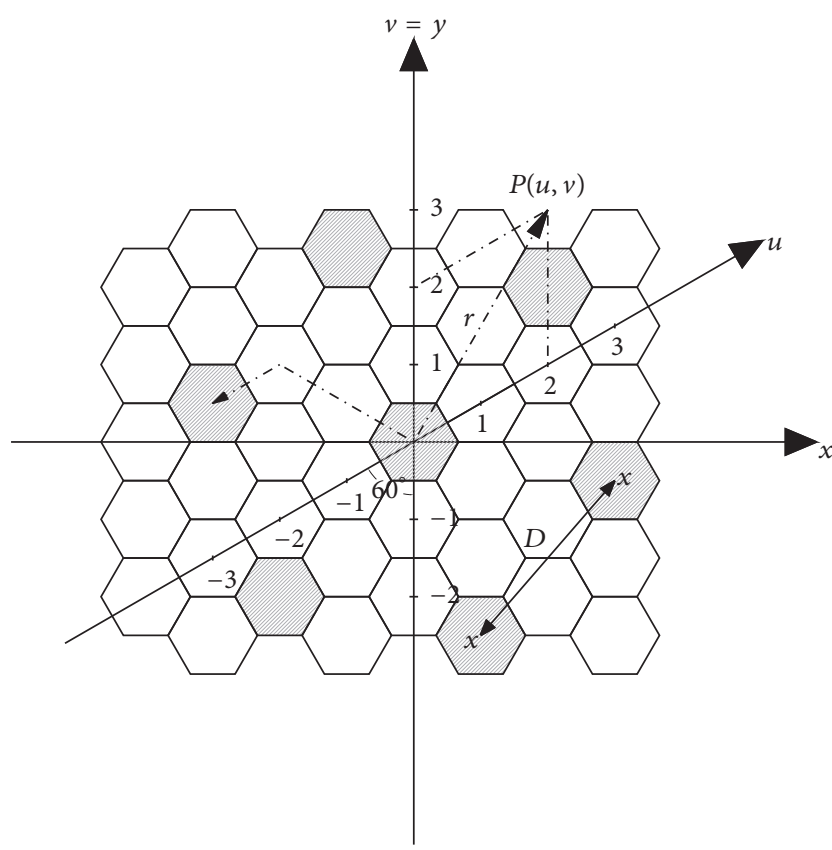

Figure 1: GSM system.

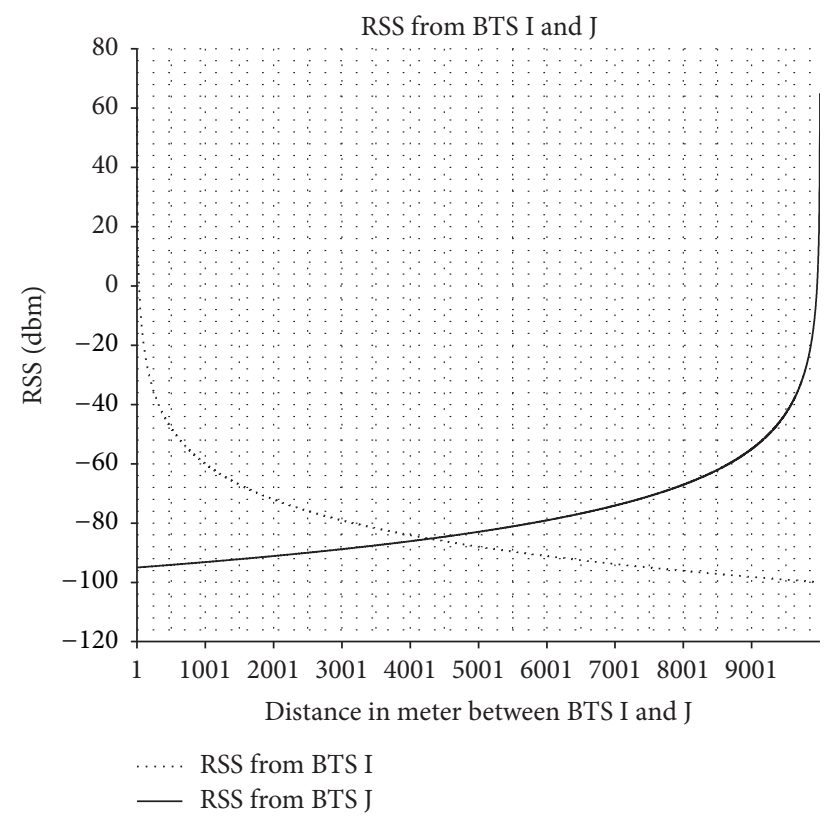

FIGURE 2: RSS from base stations I and J in $\mathrm{dbm}$.

that $4500 \mathrm{~m}$ is optimum area where handoff can take place. However, the conventional algorithm with hysteresis allows a mobile station (MS) to make handoff decision only if the RSS received from the neighboring BTS is sufficiently stronger than the current one by the specified hysteresis margin, provided a certain minimum signal level is assured.

Load balancing can be achieved with this method by handing off cell edge users in overloaded cells to migrate to underloaded neighboring BTS. But this type of load balancing is manual. Optimization engineers have to tune the hysteresis.

3.1. Path-Loss. Link budget is calculation of all the gains and losses in a transmission system. It looks at the elements that will decide the signal strength incoming at the receiver. It is necessary to determine link budget in the whole design of radio communication system. Link budget calculations are used for calculating the power levels wanted for cellular communications systems and for obtaining the BTS coverage [14].

To determine a link budget equation, it is necessary to look into all the areas where gains and losses may take place between the transmitter and the receiver. The calculation of the basic link budget is as follows.

$$
\begin{aligned}
\text { Received power }= & \text { Transmitted power }+ \text { gains } \\
& - \text { losses. }
\end{aligned}
$$

In the basic calculation of link budget equation it is assumed that the power spreads out equally in all directions from the transmitter antenna source. This is good for theoretical calculations, but not for practical calculations [14].

$$
\mathrm{Pr}=\mathrm{Pt}+\mathrm{Gt}+\mathrm{Gr}-\mathrm{LT}-\mathrm{LFS}-\mathrm{LFM}-\mathrm{LR},
$$

where

Pr is received power in $\mathrm{dbm}$,

Pt is transmitter output power in $\mathrm{dbm}$,

$\mathrm{Gt}$ is transmitter antenna gain in $\mathrm{db}$,

$\mathrm{Gr}$ is receiver antenna gain in $\mathrm{db}$,

LT is transmit feeder and associated losses (feeder, connectors, etc.) in $\mathrm{db}$,

LFS is free space loss or path-loss,

LFM is many-sided signal propagation losses (these include fading margin, polarization mismatch, and losses associated with medium through which signal is travelling),

LR is receiver feeder losses (feeder, connectors, etc.) in $\mathrm{db}$.

Figure 3 shows path-loss from two neighboring BTSs. In cellular networks, the handoff process takes on the responsibility of ensuring that any MS is always connected to the most suitable BTS.

Power budget (PBGT) handover assures that, under normal conditions, any MS is served by BTS that provides minimum path-loss and hence handoff due to path-loss takes place at about $4700 \mathrm{~m}$. However, the conventional algorithm with hysteresis allows a mobile station (MS) to make handoff decision only if the path-loss received from the neighboring BTS is better than the current one by the specified hysteresis margin, provided a certain threshold path-loss is assured. Same as in RSS based handoff algorithm, load balancing can be achieved manually by handing off cell edge users in overloaded cells to migrate to underloaded neighboring BTS. 


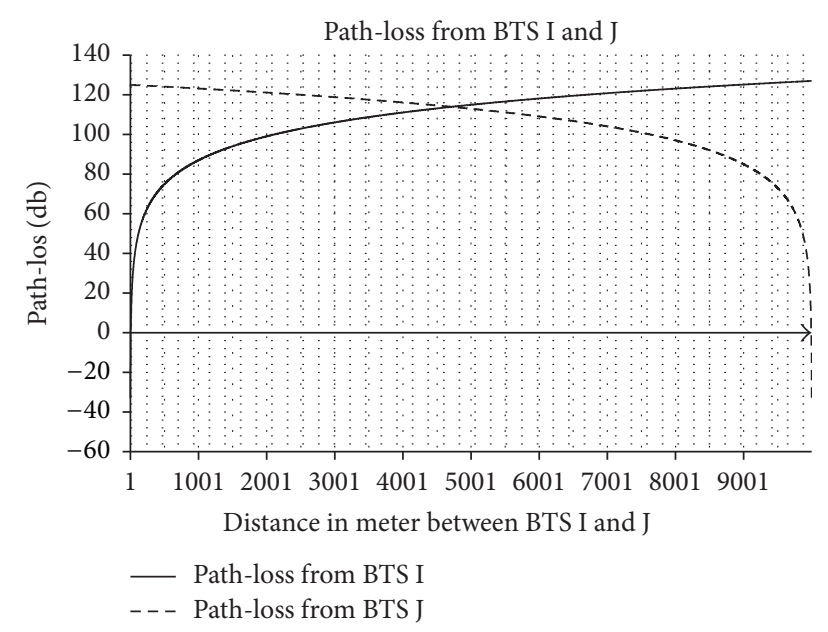

FIgURE 3: Path-loss from base stations I and $\mathrm{J}$ in $\mathrm{db}$.

\section{Signal to Noise Ratio}

It is worth mentioning that in cellular system the cochannel interference is actually the limiting factor in their efficiency and performance and not the total in-band noise in the system. This is because the unwanted signal power is very much higher than the total in-band noise (thermal, manmade) power in the system; hence the noise can be ignored [12].

Mathematically,

Signal to noise ratio, $\mathrm{SNR}=\frac{\mathrm{RSS}}{I+\mathrm{Ns}}$

Signal to interference ratio, $\frac{C}{I}=\frac{\mathrm{RSS}}{I}$ for $I \gg \mathrm{Ns}$,

where RSS is the wanted signal power, $I$ is the unwanted cochannel interfering signal power, and Ns is the total inband noise power in the system. The signal to interference ratio is a valuable measure of the performance of the modulation technique in the cellular system and it can indeed influence its spectral efficiency.

Figure 4 shows the $C / I$ from two neighboring BTSs. In cellular networks, the handoff process takes on the responsibility of ensuring that any MS is always connected to BTS having a better $C / I$. Therefore, handoff due to interference takes place at about $5000 \mathrm{~m}$ to ensure better $C / I$.

Constant hysteresis can also be introduced in interference based handoff algorithm, to fasten handoff process when BTS I is congested. This time MS on the edge of BTS I can migrate to BTS J and the load on BTS I can be decongested. If BTS $J$ is congested next time, the optimization engineers have to tune hysteresis to decongest BTS J. This task shows how load is manually shared between neighboring BTSs and it cannot be effective.

4.1. Geographical Model with Second Tier. This model, as indicted on Figure 5, is built depending on the relative geographical location of the serving and interfering BTS with respect to mobile station [12]. The model accounts for the

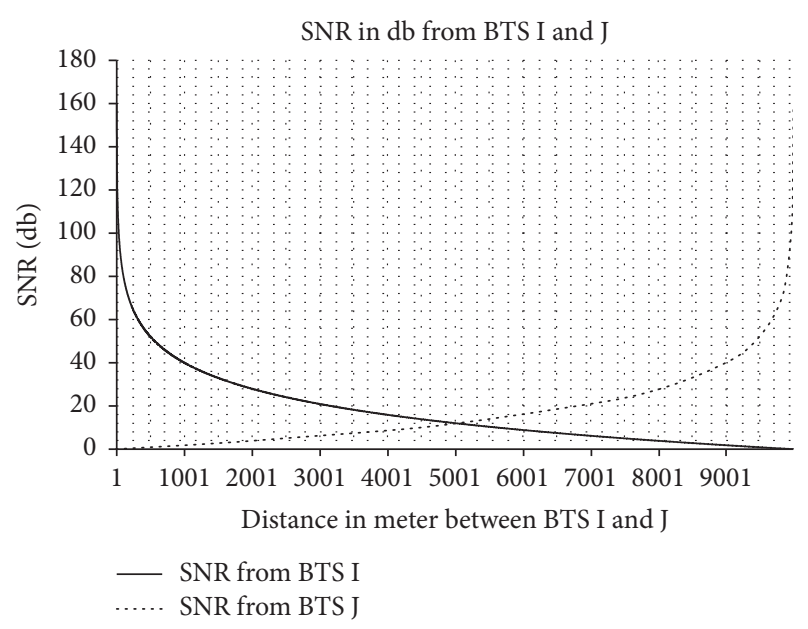

FIGURE 4: SNR from base stations I and J db.

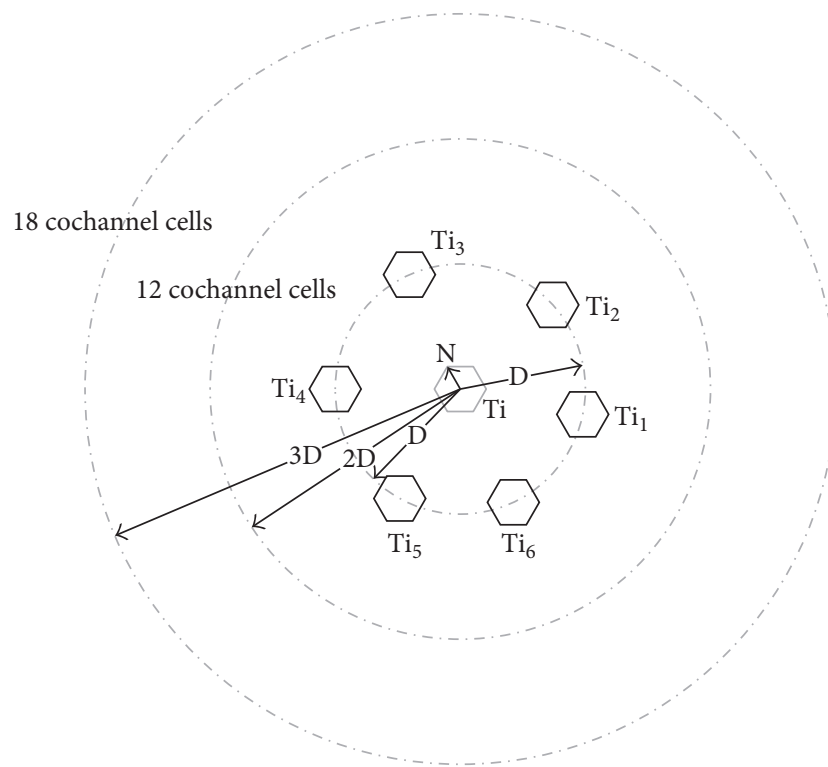

FIGURE 5: Geographical model for several tiers of interfering BTS [12].

signal path-loss due to free space and propagation loss over the flat earth.

In fully hexagon-shaped cellular system, there are always $6 \mathrm{~m}$ cochannel cells in the $m$ th tier, regardless of the number of cells per cluster. It is assumed that all cochannels interfering BTS, up to the $m$ th tier considered, are active as in a busy hour situation [12]. It is also assumed that the interference from the BTS higher order tiers (i.e., the $(m+1)$ th tier onwards) is negligible. Mobile user in the center BTS can be interfered by six BTSs on the first tier, 12 BTSs on the second tier, 18 BTSs on the third tier, and so on.

At the MS user, being served by BTS on the center of the cluster, the RSS is given by [12]

$$
\mathrm{RSS} \propto \frac{1}{R^{\alpha}},
$$


where $R$ is distance between a MS and BTS in meters and $\sigma$ path-loss constant.

The total interference from the all cochannel cells is given by [12]

$$
\begin{aligned}
& I \propto\left[\frac{6}{D^{\alpha}}+\frac{12}{(2 D)^{\alpha}}+\frac{18}{(3 D)^{\alpha}}+\frac{24}{(4 D)^{\alpha}}+\cdots\right] \\
& I \propto\left[\frac{6 \times 1}{D^{\alpha}}+\frac{6 \times 2}{(2 D)^{\alpha}}+\frac{6 \times 3}{(3 D)^{\alpha}}+\frac{6 \times 4}{(4 D)^{\alpha}}+\cdots\right]
\end{aligned}
$$

where $D$ is the frequency reuse distance in meters.

Hence,

$$
I \propto \sum_{M=1}^{T} \frac{6 m}{(m D)^{\alpha}}
$$

where $T$ is the number of tiers and $\sigma$ path-loss constant

$$
\frac{\mathrm{RSS}}{I}=\left(\frac{D}{R}\right)^{\alpha} \frac{1}{6 \sum_{m=1}^{T}\left(1 / m^{\alpha-1}\right)} .
$$

For second tier cochannel cell the above equation will reduce to the following and the third and higher tier can be ignored because (9) is Taylor series.

$$
\mathrm{SNR}=\frac{\mathrm{RSS}}{I}=\frac{(D / R)^{4}}{6.75} .
$$

In $\mathrm{db}$ the same equation will be reduced to

$$
\begin{aligned}
\mathrm{SNR} & =\frac{\mathrm{RSS}}{I} \\
& =40 \log _{10}(D)-40 \log _{10} R-10 \log _{10} 6.75 .
\end{aligned}
$$

4.2. Mathematical Justification of Geographical Model. The analytical results for propagation over a plane earth or tworay models have been derived [6]. For the BTS and mobile station elevated heights ht and hr, respectively, above the ground level and separated a distance $d$ apart, the received power Pr is given in terms of the transmitted power pt as

$$
\mathrm{RSS}=\operatorname{Pr}=\mathrm{Gt}_{\mathrm{s}} \mathrm{Gr}\left[\frac{\mathrm{ht}(\mathrm{s}) \mathrm{hr}}{R^{2}}\right]^{2} \mathrm{p}_{\mathrm{t}(\mathrm{s})} .
$$

$\operatorname{Pr}$ is the desired signal power received at the mobile station from the serving BTS, $\mathrm{p}_{\mathrm{t}(\mathrm{s})}$ is the transmitted power from the serving BTS, ht(s) is height of the serving BTS antenna in meters, hr is height of the mobile station antenna in meters, $\mathrm{Gt}_{\mathrm{s}}$ is the serving BTS antenna gain, and $\mathrm{Gr}$ is the mobile station antenna gain. $R$ is the distance between the MS and BTS in meters.

Similarly for the interfering signal power from the interfering BTS,

$$
I \approx 6.75 \mathrm{Gt}_{\mathrm{i}} \mathrm{Gr}\left[\frac{\mathrm{ht}(\mathrm{i}) \mathrm{hr}}{D^{2}}\right]^{2} \mathrm{p}_{\mathrm{t}(\mathrm{i})} .
$$

$I$ is the unwanted signal power received at the mobile station from the interfering BTS, $\mathrm{p}_{\mathrm{t}(\mathrm{i})}$ is the transmitted power from the interfering BTS, ht(i) is height of the interfering BTS antenna in meters, hr is height of the mobile station antenna in meters, $\mathrm{Gt}_{\mathrm{i}}$ is the interfering BTS antenna gain, $\mathrm{Gr}$ is the mobile station antenna gain, and $D$ is the frequency reuse distance in meters.

Hence combining equations

$$
\frac{\mathrm{RSS}}{I}=\mathrm{SNR}=\frac{\mathrm{Gt}_{\mathrm{s}} \mathrm{Gr}\left[\mathrm{ht}(\mathrm{s}) \mathrm{hr} / d^{2}\right]^{2} \mathrm{p}_{\mathrm{t}(\mathrm{s})}}{6.75 \mathrm{Gt}_{\mathrm{i}} \mathrm{Gr}\left[\mathrm{ht}(\mathrm{i}) \mathrm{hr} / d^{2}\right]^{2} \mathrm{p}_{\mathrm{t}(\mathrm{i})}}
$$

From (14), it can be shown that SNR can be maximized by maximizing $\mathrm{Gt}_{\mathrm{s}}, \mathrm{h}_{\mathrm{ts}}$, and $\mathrm{p}_{\mathrm{t}(\mathrm{s})}$.

$$
\mathrm{SNR}=\frac{\mathrm{RSS}}{I}=\frac{(D / R)^{4}}{6.75} \text { same as }(10) .
$$

Equation (15) agrees with (10) based on the assumption of equal radiated power in the entire clusters; nevertheless (10) remains valid for mixed cell size when $\mathrm{Gt}_{\mathrm{s}} \neq \mathrm{Gt}_{\mathrm{i}}, \mathrm{p}_{\mathrm{t}(\mathrm{s})} \neq$ $\mathrm{p}_{\mathrm{t}(\mathrm{i})}, \mathrm{Gt}_{\mathrm{s}} \neq \mathrm{Gt}_{\mathrm{i}}$, and ht(s) $\neq \mathrm{ht}(\mathrm{i})$.

\section{Simulation Using Matlab}

5.1. Fuzzification. In the first step of the handoff process, the model would collect the parameters like RSS, path-loss, and SNR and traffic load of the base station and fed into a fuzzifier. The fuzzifier transforms real time measurements into fuzzy sets. In order to improve the reliability and robustness of the system, Gaussian membership functions (MFs) are used as an alternative to the traditional triangular MFS [15]. For instance, if RSS is considered in crisp set, it can only be weak or strong. RSS cannot be both at a time. However, in a fuzzy set the signal can be considered as weak signal and medium at the same time with graded membership. The membership values are obtained by mapping the values obtained for particular parameter into a membership function.

5.2. Fuzzy Inference. The second step of handoff process involves feeding the fuzzy sets into an inference engine, where a set of fuzzy IF-THEN rules are applied to obtain fuzzy decision sets [15]. These sets are mapped to the corresponding Gaussian membership functions. Since there are four fuzzy inputs and each of them has three subsets, there are $3^{4}=81$ rules (e.g., if RSS is strong and SNR is high and path-loss is small and load is low, then there is no handoff). Fuzzy rules can be defined as a set of possible scenarios. For simple understanding, the set (no handoff, wait, be careful, and handoff) is used to represent the fuzzy set of output handoff decision; the range of the decision matrix is from 0 to 1 , where 0 is no handoff and 1 is exactly handoff.

5.3. Defuzzification. Finally, the output fuzzy decision sets are aggregated into a single fuzzy set and passed to the defuzzifier to be converted into a precise quantity during the last stage of the handoff decision. The centroid of area method is elected to defuzzify for changing the fuzzy value into the crisp set [11]. Figure 6 shows the structure of the proposed algorithm, with four inputs and one output, fuzzifier and defuzzifier. 


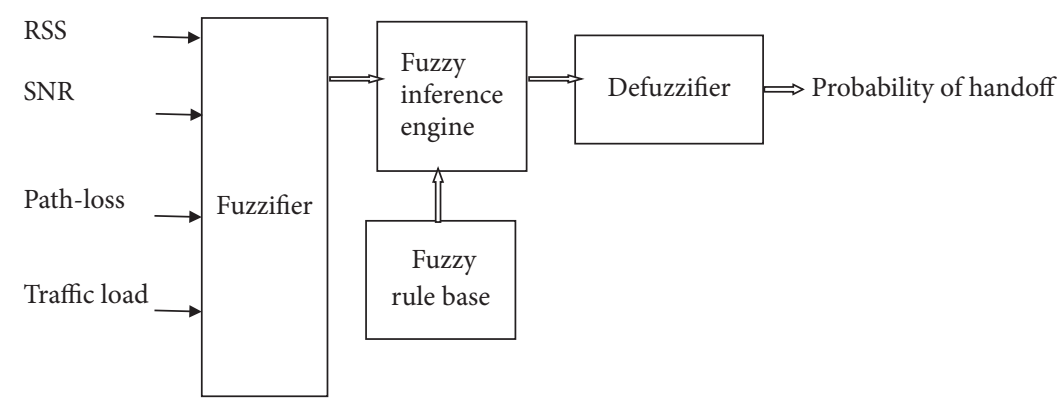

FIGURE 6: Structure of the proposed algorithm.

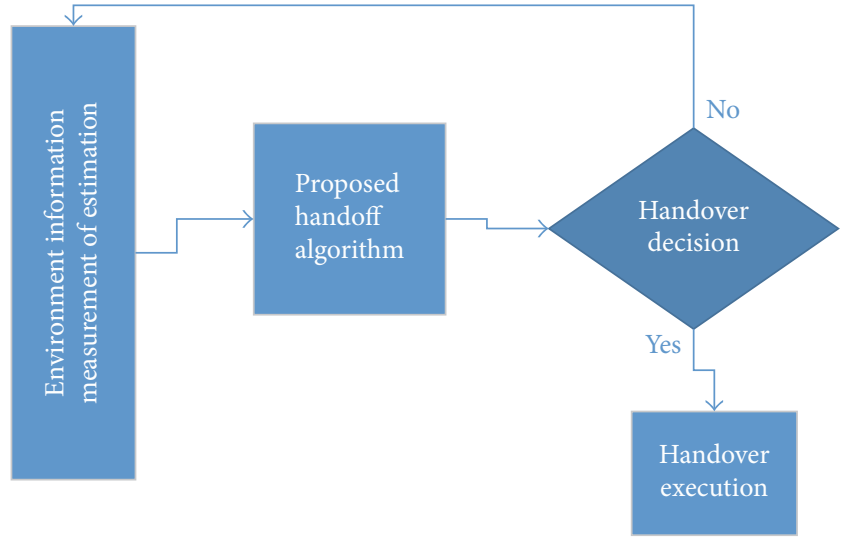

FIGURE 7: General structure of the proposed multicriteria handoff algorithm.

For system simulation Mamdani Fuzzy Inference system is proposed due to fact that Mamdani method is well suited to human input and the nature of wireless is nonlinear [15]. Fuzzy inferences gather the input values of RSS and pathloss and SNR are collected from the propagation model and the load of the BTS from BTS Controller (BSC) and then evaluate them according to the fuzzy interference rules base. The composed and aggregated output of rules evaluation is defuzzified using the centroid of area method and crisp output is obtained [11].

\section{Multicriteria Handoff Algorithm}

As indicated in Figure 7, fuzzy logic reasoning scheme is applied for mapping of nonlinear data set to scalar output figure. When the problems are in doubt and ambiguous, then to anticipate the correct value among all uncertainties we select fuzzy logic to develop computational techniques that can carry out reasoning and problem solving tasks that require human intelligence [16]. Thus, fuzzy logic is used to choose the optimal serving BTS among given neighboring BTSs for handoff decision founded on the multiple parameters (RSS, path-loss, $C / I$, and the load of the BTS) as crisp inputs and give the best possible answer to choose the best BTS.

Using single criteria may result in inefficient handover and uneven traffic load distribution. To decide handoff

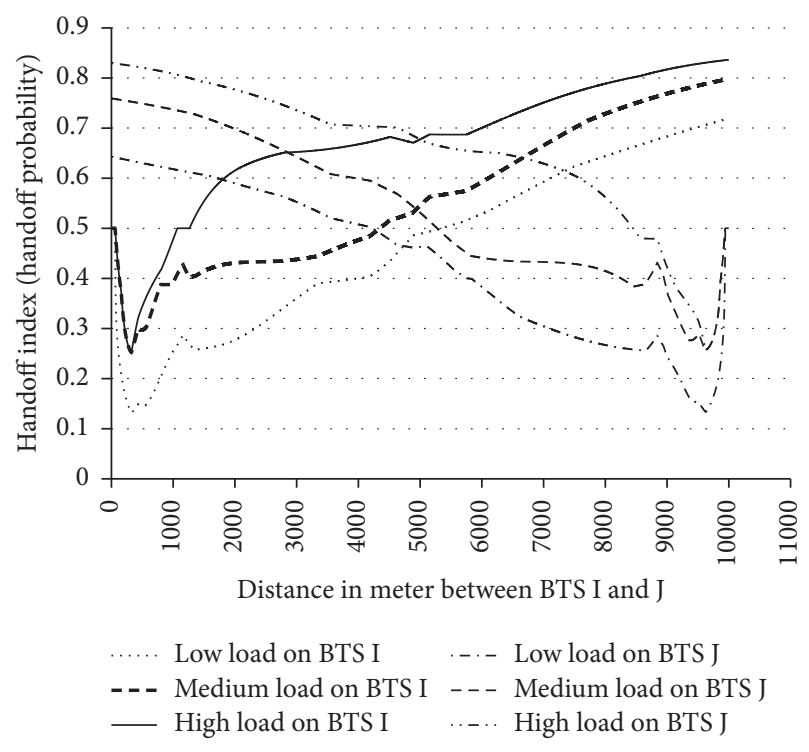

FIGURE 8: Movement of the mobile station between two adjacent BTSs I and J.

more accurately, more parameters are needed [15]. In our study, we propose four criteria to decide handoff. These input parameters are RSS, path-loss, $C / I$, and traffic load of the BTS. The first three criteria are user mobility related parameters; meanwhile traffic load on the BTS is networkrelated parameter [15].

Mobility related parameters are gathered from the wireless environments using propagation models and fed into the fuzzy system along with the traffic load of the BTS [15].

The proposed handoff algorithm gives the handoff index of the serving BTS and all the neighboring BTS and the handoff process are executed once the neighboring BTS is identified better. The output of the fuzzy system is between 0 and 1 , where 0 is no handoff and 1 is exactly handoff; the lower the better for MS to be served [15].

\section{Result and Discussion}

Figure 8 shows a mobile station is moving from one BTS (named BTS I) to another BTS (named BTS J). The handoff index of BTS I increases as a mobile station moves away and 
decreases as mobile station gets close to the BTS J. Under normal condition the BTS I serves the first $5000 \mathrm{~m}$ and BTS J serves the second $5000 \mathrm{~m}$. The handoff index varies depending on the load BTS. The higher the load of BTS the higher the handoff index of the BTS so that the handoff process takes place very fast. For low and medium load on the BTS, the handoff index will be smaller so that the handoff process will be delayed.

The fastest handoff takes place at about $1700 \mathrm{~m}$ when BTS I has high load and BTS J has low load, so that the coverage area of a BTS I can virtually be shrunk inwards and mobile on that region is served by the neighboring BTS J. This technique is called dynamic mobility load balancing. The logic behind this method is to adjust the handoff regions by biasing the handoff region depending on the load of the BTSs, causing cell edge users in loaded cells to migrate to less loaded overlapping cells, thereby releasing some traffic channels being occupied by edge user so that the call can proceed. With this process new call blocking can be solved in case the neighboring BTS has low load, satisfying the minimum RSS required. If the neighboring BTS has high load then the handoff takes place on the ideal boundary of the BTS as the conventional handoff as shown in Figure 8.

The most delayed handoff takes place at about $7400 \mathrm{~m}$ when the BTS I has low load and BTS J has high load. In this case BTS I coverage area can virtually be expanded towards loaded BTS J. This is due to fact that if no channel is available in the BTS J, the handoff call is blocked which leads a call to terminate prematurely due to dropout. The MS keeps on BTS I as long as RSS, path-loss, and SNR of BTS I are on the recommended threshold. The is a method by which handoff blocking is reduced and avoids call drop due to handover failure as there is no channel available in BTS J [17]. If the neighboring BTS has also low load, then there is no need to delay handoff process. Handoff will take place on the ideal boundary between the base stations.

Figure 3 shows how the mobile in the region between $1700 \mathrm{~m}$ and $7400 \mathrm{~m}$ swings between BTSs I and J dynamically depending on the load on the BTS, such that the coverage area of a BTS can dynamically be expanded towards a nearby loaded cell or shrunk towards cell center for a loaded sector. Therefore, this mechanism activates a handoff procedure to shift some traffic of a loaded cell towards a lightly loaded cell.

For the same load on either BTS the multicriteria have the same performance as single criteria (RSS based, pathloss based, and SNR based) handoff algorithm; the handoff process takes place at an ideal boundary between the BTSs which is $5000 \mathrm{~m}$.

\section{Conclusion}

In this paper, we show that it is possible to balance traffic load of cellular network by handing off some ongoing calls on cell edge in highly loaded cells to migrate to overlapping underloaded cells, such that the coverage area of loaded BTS virtually shrunk towards cell center of a loaded sector. In case of low load scenarios, the coverage area of a BTS is presumed to be virtually widened to cover up to the partial serving area of neighboring BTS.

\section{Conflicts of Interest}

The authors declare that they have no conflicts of interest.

\section{References}

[1] K. Raymond, R. Arnott, R. R. Trivisonno, and M. Kubota, "On mobility load balancing for LTE systems," in Proceedings of the IEEE 72nd Vehicular Technology Conference Fall, IEEE, September 2010.

[2] S. Scellato, L. Fortuna, M. Frasca, J. Gómez-Gardeñes, and V. Latora, "Traffic optimization in transport networks based on local routing," The European Physical Journal B, vol. 73, no. 2, pp. 303-308, 2010.

[3] P. Muñoz, R. Barco, I. De La Bandera, M. Toril, and S. Luna-Ramírez, "Optimization of a fuzzy logic controller for handover-based load balancing," in Proceedings of the IEEE 73rd Vehicular Technology Conference, IEEE, May 2011.

[4] K. A. Akpado, O. S. Oguejiofor, C. O. Ezeagwu, and A. U. Okolibe, "Investigating the impacts of BTS antenna height, tilt and transmitter power on network coverage," International Journal of Engineering Science Invention, vol. 2, no. 7, pp. 32-38, 2013.

[5] K. A. Ali, H. S. Hassanein, and H. T. Mouftah, "Directional cell breathing based reactive congestion control in WCDMA cellular networks," in Proceedings of the 12th IEEE International Symposium on Computers and Communications, pp. 685-690, July 2007.

[6] A. Goldsmith, Wireless Communications, Cambridge University Press, New York, NY, USA, 1st edition, 2005.

[7] S. Srinivas, A. Sahu, and S. K. Jena, "Efficient load balancing in cloud computing using fuzzy logic," IOSR Journal of Engineering (IOSRJEN), vol. 2, pp. 65-71, 2012.

[8] Chandrasekhar and P. K. Behera, "Use of adaptive resonance theory for vertical handoff decision in heterogeneous wireless environment," International Journal of Recent Trends in Engineering, vol. 2, no. 3, 2009.

[9] R. Nasri and Z. Altman, "Handoff adaptation for dynamic load balancing in 3GPP long term evolution systems," in Proceedings of the International Conference on Advancnes in Mobile Computing Multimedia (MoMM '07), 2007.

[10] F. Kaleem, A. Mehbodniya, K. K. Yen, and F. Adachi, "A fuzzy preprocessing module for optimizing the access network selection in wireless networks," Advances in Fuzzy Systems, vol. 2013, Article ID 232198, 9 pages, 2013.

[11] F. Kaleem, VHITS: Vertical Handoff Initiation and Target Selection in a Heterogeneous Wireless Network, Florida International University, 2012.

[12] H. Hammunda, Cellular Mobile Radio Systems: Designing Systems for Capacity Optimization, John Wiley and Sons' Ltd, 1997.

[13] L. Chandra Paul, "Handoff/handover mechanism for mobility improvement in the wireless," The Global Journal of Researches in Engineering, vol. 13, 2013.

[14] P. K. Sharma and R. K. Singh, "Cell coverage area and link budget calculations in GSM system," International Journal of Modern Engineering Research (IJMER), vol. 2, no. 2, pp. 170-176, 2012.

[15] T. G. Solomon, D. B. O. Konditi, and E. N. Ndungu, "Real time traffic balancing in cellular network by multi-criteria handoff algorithm using fuzzy logic," International Journal of Emerging Technology and Advanced Engineering, vol. 4, no. 4, 2014. 
[16] G. P. Pollini, “Trends in Handover design,” IEEE Communications Magazine, vol. 34, no. 3, pp. 82-90, 1996.

[17] Y. Iraqi and R. Boutaba, "handoff and call dropping probabilities in wireless cellular networks," in Proceedings of the International Conference on Wireless Networks, Communications and Mobile Computing, June 2005. 

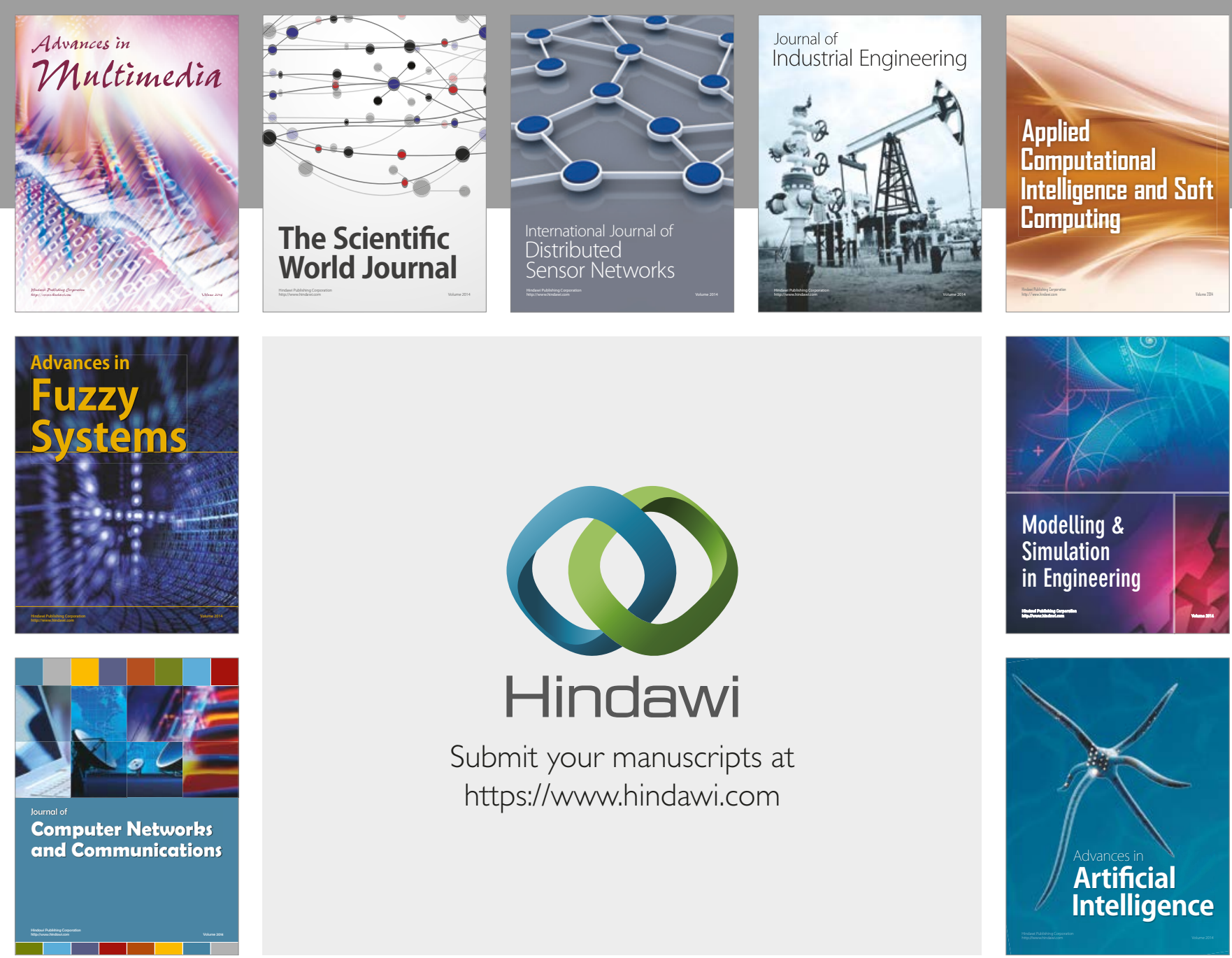

\section{Hindawi}

Submit your manuscripts at

https://www.hindawi.com
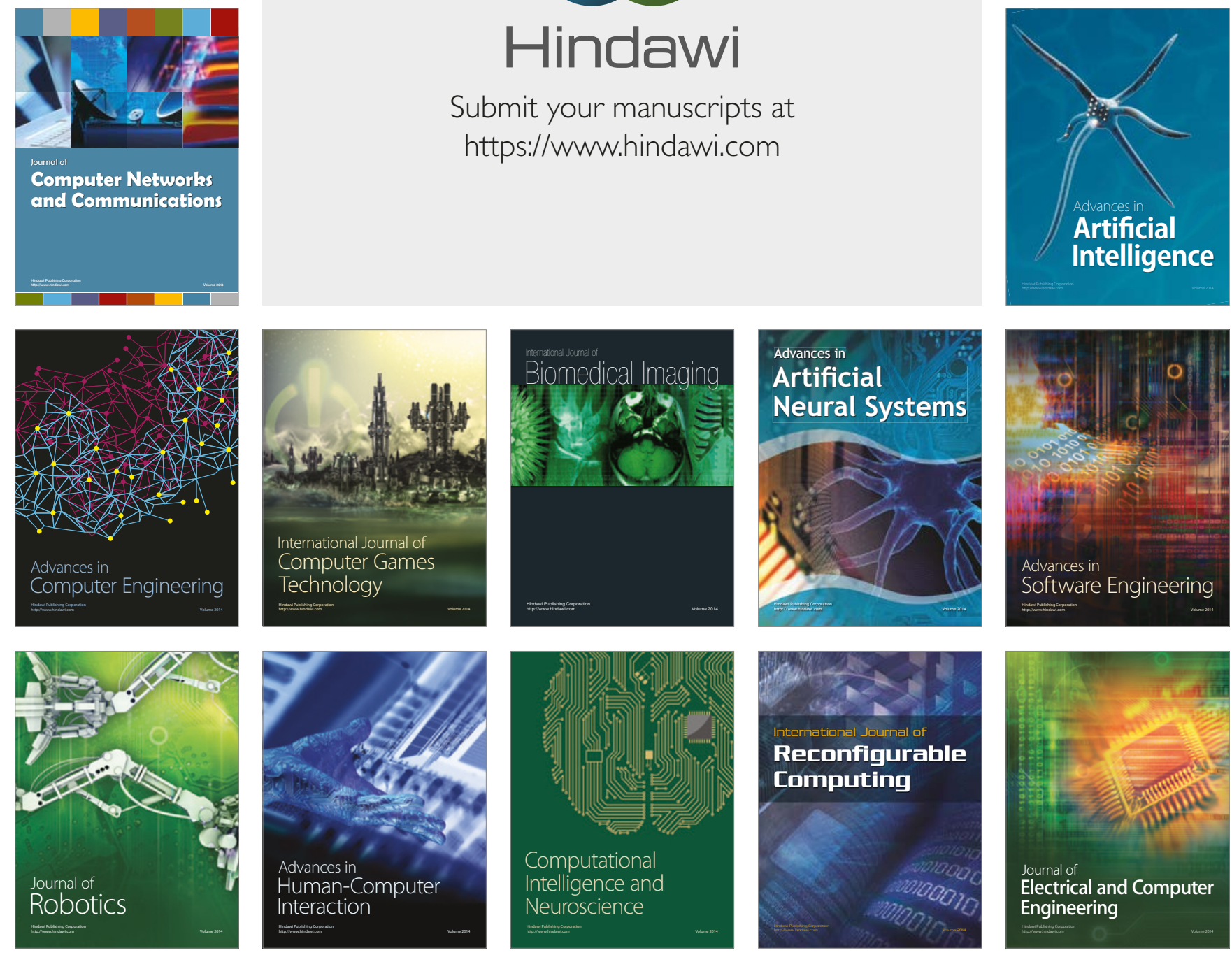